\title{
Two-Dimensional Impulsively Stimulated Resonant Raman Spectroscopy of Molecular Excited States
}

\author{
Giuseppe Fumero๑, ${ }^{1,2,}$ Christoph Schnedermann $\odot,{ }^{3,4, \ddagger}$ Giovanni Batignani®${ }^{1},{ }^{1}$ Torsten Wende, ${ }^{3}$ Matz Liebel@, ${ }^{3,5}$ \\ Giovanni Bassolino, ${ }^{3}$ Carino Ferrante, ${ }^{1,6}$ Shaul Mukamel@ ${ }^{7}$ Philipp Kukura $\odot^{3, *}$ and Tullio Scopigno $\oplus^{1,6, \dagger}$ \\ ${ }^{1}$ Dipartimento di Fisica, Sapienza Universitá di Roma, Piazzale Aldo Moro 5, Roma, I-00185, Italy \\ ${ }^{2}$ Dipartimento di Scienze di Base e Applicate per l'Ingegneria, Sapienza Universitá di Roma, \\ Via Antonio Scarpa 14/16, Roma, I-00161, Italy \\ ${ }^{3}$ Physical and Theoretical Chemistry Laboratory, South Parks Road, Oxford, OX1 3QZ, United Kingdom \\ ${ }^{4}$ Cavendish Laboratory, University of Cambridge, J. J. Thomson Avenue, \\ Cambridge CB3 OHE, United Kingdom \\ ${ }^{5}$ ICFO -Institut de Ciencies Fotoniques, The Barcelona Institute of Science and Technology, \\ Barcelona, 08860 Castelldefels, Spain \\ ${ }^{6}$ Istituto Italiano di Tecnologia, Center for Life Nano Science @ Sapienza, Roma, I-00161, Italy \\ ${ }^{7}$ Department of Chemistry and Physics and Astronomy, University of California, \\ Irvine, California 92697, USA
}

(Received 26 January 2019; revised manuscript received 19 October 2019; accepted 3 December 2019; published 28 February 2020)

\begin{abstract}
Monitoring the interactions between electronic and vibrational degrees of freedom in molecules is critical to our understanding of their structural dynamics. This is typically hampered by the lack of spectroscopic probes able to detect different energy scales with high temporal and frequency resolution. Coherent Raman spectroscopy can combine the capabilities of multidimensional spectroscopy with structural sensitivity at ultrafast timescales. Here, we develop a three-color-based 2D impulsive stimulated Raman technique that can selectively probe vibrational mode couplings between different active sites in molecules by taking advantage of resonance Raman enhancement. Three temporally delayed pulses generate nuclear wave packets whose evolution reports on the underlying potential energy surface, which we decipher using a diagrammatic approach enabling us to assign the origin of the spectroscopic signatures. We benchmark the method by revealing vibronic couplings in the ultrafast dynamics following photoexcitation of the green fluorescent protein.
\end{abstract}

DOI: 10.1103/PhysRevX.10.011051

Subject Areas: Atomic and Molecular Physics, Optics, Physical Chemistry

\section{INTRODUCTION}

The investigation of light-induced processes is essential to the understanding of a variety of complex phenomena at the interface between physics, chemistry, and biology, in which excited-state dynamics cause the transient reconfiguration of atomic positions and electronic phases. Following the absorption of a photon, the behavior of the system is dictated by the interplay between vibrational and electronic degrees of freedom of the system and

\footnotetext{
philipp.kukura@chem.ox.ac.uk

†tullio.scopigno@uniroma1.it

${ }^{\ddagger}$ These authors contributed equally.
}

Published by the American Physical Society under the terms of the Creative Commons Attribution 4.0 International license. Further distribution of this work must maintain attribution to the author(s) and the published article's title, journal citation, and DOI. the environment [1-4]. The evolution typically occurs along a multidimensional landscape, which is effectively represented by vibrationally structured potential energy surfaces (PESs) and determines the competition between all the available radiative and nonradiative relaxation channels.

Ultrafast spectroscopy exploits tailored sequences of laser pulses to photoexcite and subsequently probe these channels, with the aim of unveiling the dynamics and the underlying vibronic structure. This goal requires the correct identification of the excited-state PESs involved in the photoinduced process as well as the mapping of their relative orientations and displacements. A number of different pulse schemes and strategies have been developed to meet these tasks, but the unambiguous identification of vibronic properties, such as quasiparticle couplings, mode-mixing and nonadiabatic effects, remains challenging $[5,6]$ - in particular, on electronically excited states after the system has left the Franck-Condon (FC) regiondue to overlapping signal contributions arising from 
different physical processes. Linear vibrational techniques, such as infrared and spontaneous Raman spectroscopy, cannot monitor vibrational coherences on the excited states, whereas UV-visible absorption spectroscopy usually lacks the desired structural sensitivity. Considerable efforts have been aimed at the development of multidimensional spectroscopic techniques in order to separate the photoinduced response over additional spectral dimensions [7-19], which arise from the parameters tunable during the experiment, such as the time delays between multiple excitation pulses. The correlations of features on different dimensions offer a different perspective on the vibrational landscape, providing a connection between the structure of the system and its dynamics.

Coherent multidimensional Raman spectroscopy is well suited to directly address excited-state vibrational properties in time and frequency domains due to its "fingerprint" specificity over the full vibrational manifold of interest [20-24]. Furthermore, Raman spectroscopy benefits from resonance enhancements of specific chromophore signatures and thereby provides a route to comprehensively investigate vibrational energy flow during reactive transformations by selectively probing specific environments [25]. In particular, time-domain impulsive stimulated Raman scattering (ISRS) [26-36] offers several advantages over its frequency-domain analogues for the detection of vibronic features, especially for low-frequency modes, by efficiently removing elastic scattering contributions and background noise [37-40]. Its multidimensional extension, 2D-ISRS, has been theoretically proposed initially [41] and realized in both nonresonant [42,43] and resonant $[44,45]$ implementations to study ground-state intramolecular vibrational anharmonicities, nonlinear corrections to the molecular polarizability, product-reactant correlations, and solvation dynamics, up to the recent realization of single-pulse 2D spectroscopy by means of appropriately shaped light pulses [46], with possible applications theoretically suggested for the x-ray domain [47].

The development of a 2D-ISRS scheme aimed to probe electronically excited-state vibrations would disclose the conformation of excited energy landscapes, providing direct information on specific molecular properties, such as the geometrical configuration and orientations of different PESs. This information is encoded in the harmonic fifth-order Raman response, which vanishes in the off-resonant regime. Critically, resonant 2D-ISRS demands a substantial increase in complexity, regarding both the experimental layout [48] and the data interpretation, in order to establish a protocol able to disentangle the genuine excited-state contributions from ground-state features and to assign the measured $2 \mathrm{D}$ peaks to the corresponding molecular origin.

Here, we address these challenges by presenting resonant excited-state 2D-ISRS. In striking contrast with the time-resolved ISRS approach-which employs a photopump to create an electronically excited-state population and monitors the temporal evolution of vibrational frequencies during a photoreaction $[30,32,49]$-by exploiting three femtosecond pulses for stimulating Raman coherences, we induce and probe vibronic correlations on the electronically excited manifold. Building on the resonance Raman enhancement, in our realization we tune the optical wavelengths of the pulses used in 2D-ISRS in resonance with the static and transient electronic absorption transitions to isolate contributions pertaining to a targeted electronic state. Importantly, our pulse configuration additionally suppresses undesired lower-order cascade effects, which typically mask the 2D Raman features [50,51].

We decipher the complex multidimensional response by developing an analytical method to read out the properties of vibronic coupling, which allows us to map out the multidimensional PES from the intensities and locations of diagonal and off-diagonal peaks in the 2D-ISRS spectra. We subsequently apply our approach to study the wild-type green fluorescent protein (GFP) [52,53], a prototypical system used in the fluorescence bioimaging community, during its initial photoinduced relaxation process. By collecting 2D-ISRS spectra over the full vibrational fingerprint region, we project the initially induced coherences on a separate temporal dimension and then probe their correlations as they evolve out of the FC region. We rationalize our experimental results by means of a harmonic treatment of the molecular Hamiltonian, demonstrating that this simple model is able to trace the origin of the different couplings. We further illustrate how a careful design of the experimental conditions enables the observation of dark or weak vibrational modes as coupling peaks, PES displacements along normal coordinates, and signatures of harmonic mode mixing in the excited states beyond the approximation of linearly displaced potentials.

\section{RESULTS}

\section{A. Three-color 2D-ISRS technique}

The pulse sequence of three-color 2D-ISRS, along with an illustrative sketch of its working principle, is presented in Figs. 1(a) and 1(b), respectively. A femtosecond, frequency-tunable actinic pulse $\mathcal{E}_{a}$, resonant with the $\mathrm{S}_{0}-\mathrm{S}_{1}$ absorption maximum, promotes the system into an excited electronic state $S_{1}$, and it impulsively generates vibrational coherences due to its broad spectral bandwidth, provided that the pulse duration is shorter than the vibrational period [27]. Following a variable time delay $\mathrm{T}_{1}$, a femtosecond impulsive Raman pulse $\mathcal{E}_{p}$, resonant with the $\mathrm{S}_{1}-\mathrm{S}_{n}$ excited-state absorption, induces additional vibrational coherences on $S_{1}$. After a second variable time delay $\mathrm{T}_{2}$, a broadband white-light continuum (WLC) probe pulse records the temporal evolution of the vibrational coherences through spectrally-resolved transient absorption. The nonlinear polarization induced by the actinic and Raman 
(a)

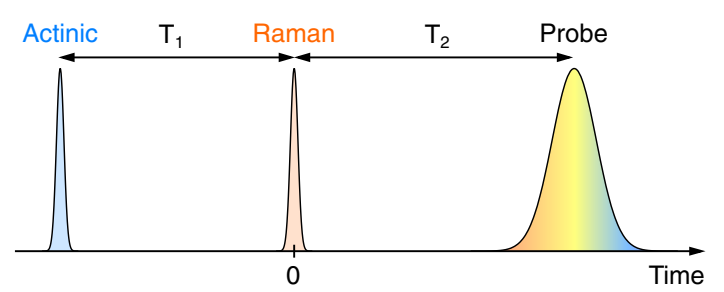

(b)

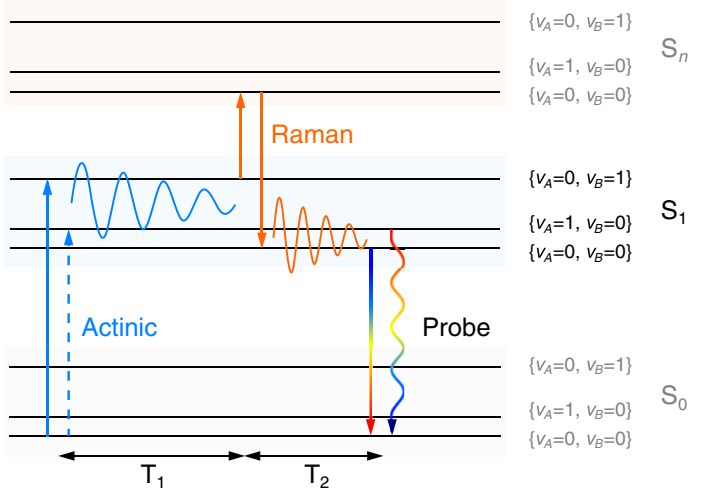

(c)

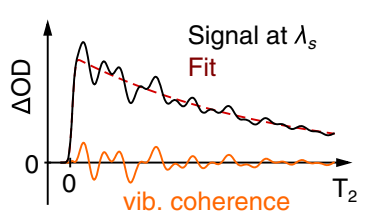

(d)

(e)
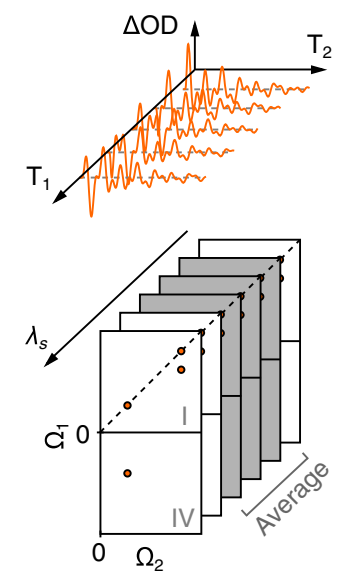

FIG. 1. Concept of 2D-ISRS. (a) Pulse sequence used for the experiment. (b) Energy ladder scheme illustrating an example of signal generation. Horizontal black lines represent the vibrational levels of the sample organized in three electronic manifolds. Dotted and solid arrows indicate light interactions with the ket and the bra side of the density matrix representing the state of the system. (c)-(e) Overview of the data analysis required in 2D-ISRS. (c) Transient absorption signals recorded along $\mathrm{T}_{2}$ for a given $\mathrm{T}_{1}$ over all probe wavelengths $\lambda_{s}$, and the vibrational coherence contribution, which is isolated by fitting and subtracting the electronic background. (d) At each $\lambda_{s}$, we collect the coherence for each $\mathrm{T}_{1}$ to build $S\left(T_{1}, T_{2}\right)$, which is (e) subsequently $2 \mathrm{D}$ Fourier transformed to yield $S\left(\Omega_{1}, \Omega_{2}\right)$, after averaging the WLC probe wavelengths $\lambda_{s}$ along the desired resonance region.

pulses oscillates during $T_{1}$ and $T_{2}$ at the frequencies of the stimulated Raman-active modes, thereby modulating the transmitted broadband probe pulse. The spectral content of these coherent oscillations is retrieved via a $2 \mathrm{D}$ Fourier transformation along $\mathrm{T}_{1}$ and $\mathrm{T}_{2}$, which reveals correlations between Raman modes appearing as cross and combination peaks. By tuning the actinic and Raman pulses into resonance with different excited-state transitions and selecting the probe wavelength region corresponding to the stimulated emission, the proposed scheme allows us to select $\mathrm{S}_{1}$ as the PES on which the vibrational coherences are created and probed. We refer the reader to Fig. S2 of the Supplemental Material (SM) [54], where we report on the modification of the 2D-ISRS map upon changing the resonance condition of the probe pulse.

Specifically, we extract the oscillatory part of the detected signal at each probe wavelength along $T_{2}$ for every recorded point in time along $\mathrm{T}_{1}$, which yields the signal $S\left(T_{1}, T_{2}\right)$ [Figs. 1(c) and 1(d)]. Note that $S\left(T_{1}, T_{2}\right)$ (reported in Fig. S1 of the SM [54]) is subsequently 2D Fourier transformed and averaged over the spectral region of interest to provide the final 2D-ISRS map $S\left(\Omega_{1}, \Omega_{2}\right)$ [Fig. 1(e); see the Appendix $\mathrm{C}$ for further details]. Considering that the 2D Fourier transform of a real time-domain signal is centrosymmetric, we present only the first and fourth spectral quadrants, which are associated with signal components of the same and the opposite sign along the two frequency axes, respectively [55].

Importantly, since the Raman pulse must interact with a molecule that was previously excited by the actinic pulse in order to contribute to the excited-state 2D-ISRS signal, the resonant scheme renders our technique free from lower-order background involving cascades between two different molecules that generally affect fifth-order spectroscopies [50,51,56,57].

\section{B. Experimental measurements of wild-type GFP}

To explore the capabilities of our technique, we measured resonant 2D-ISRS spectra of wild-type GFP during the early stages of its photodynamics. Under physiological conditions, the GFP chromophore exists predominantly in a neutral, protonated $\mathrm{A}_{0}$ form [Fig. 2(a), gray structure]. Photoexcitation at about $397 \mathrm{~nm}$ [Fig. 2(b), gray absorption spectrum] promotes the chromophore to the first excited state $A^{*}$ [Fig. 2(a), blue structure], which subsequently undergoes an excited-state proton transfer (ESPT) of the phenolic proton from Tyr 66 to Glu 222, across a hydrogen bonding network to yield the highly fluorescent, deprotonated I* form [Fig. 2(a), green structure] [58].

We focus on the rapid initial $(\sim 1 \mathrm{ps})$ relaxation out of the FC region, which precedes ESPT, occurring on a longer timescale $(2-10 \mathrm{ps})[59,60]$. As the photoinduced evolution 
(a)

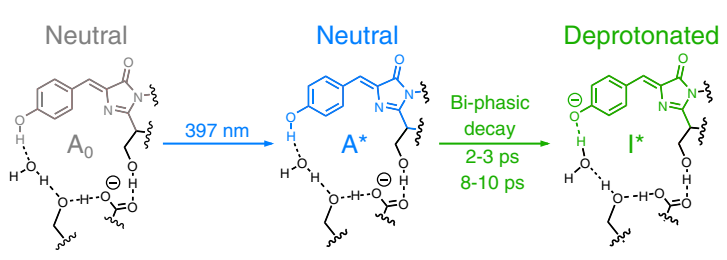

(b)

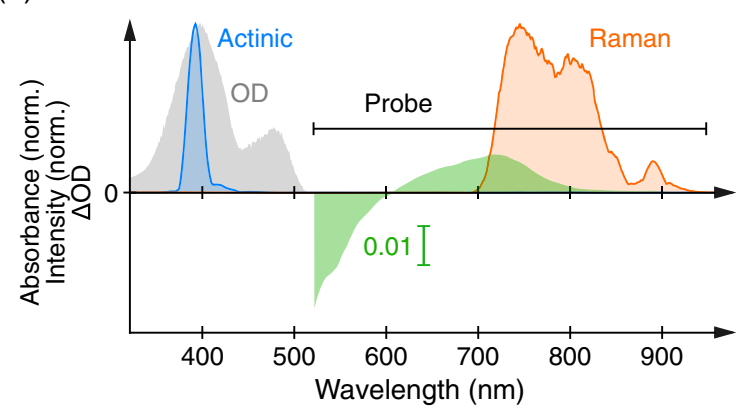

(c)

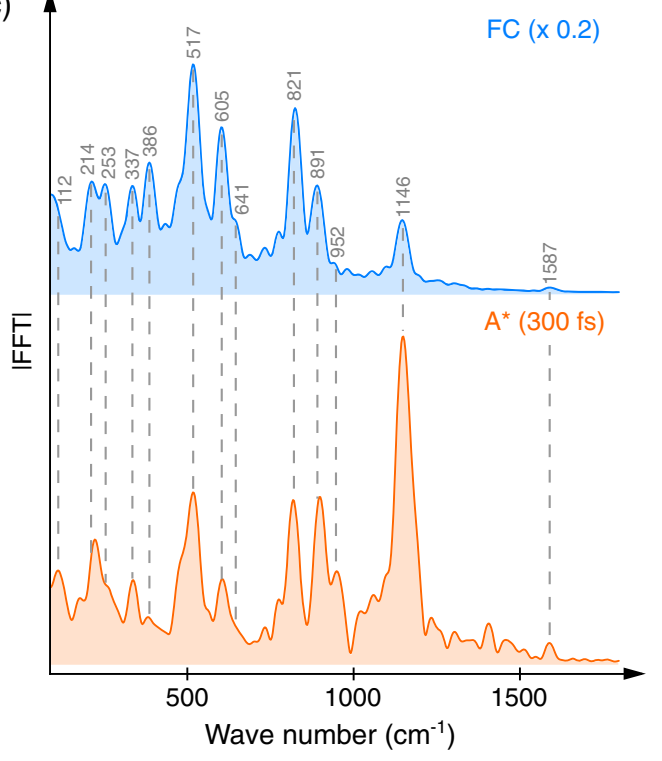

FIG. 2. Experimental characterization of wild-type GFP. (a) Photodynamics of GFP. Photoexcitation of the neutral $\mathrm{A}_{0}$ chromophore at $397 \mathrm{~nm}$ prepares $\mathrm{A}^{*}$, which decays with biphasic dynamics (2-3 and 8-10 ps) to the fluorescently active deprotonated I* chromophore $[59,60]$. (b) Normalized absorption spectrum (gray) and transient absorption spectrum at $1 \mathrm{ps}$ (green) and employed actinic and Raman pulse spectra (blue and orange). (c) Comparison of FC spectrum (blue) and $\mathrm{A}^{*}$ Fourier amplitude spectrum binned in $\mathrm{T}_{1}$ to 300 fs to average out any effect of oscillatory modulations along $\mathrm{T}_{1}$ (orange). The absolute amplitude of the FC spectrum was scaled by 0.2 for clarity. Raman spectra were averaged over a probe wavelength region from 575 to $615 \mathrm{~nm}$ (stimulated emission), and coherent oscillations were Fourier transformed over a time delay of 1 ps (see Appendix C for further details).

is accompanied by a large Stokes shift, the transient absorption spectrum after 1 ps from photoexcitation at $397 \mathrm{~nm}$ exhibits the characteristic stimulated emission band at $509 \mathrm{~nm}$ known for GFP, along with a broad photoinduced absorption band at higher wavelengths [720 nm, Fig. 2(b), green spectrum]. Beyond transient absorption, we perform 1D- and 2D-ISRS measurements on GFP using a 15-fs actinic pulse in combination with a 9-fs Raman pump pulse, whose spectral envelopes are shown in Fig. 2(b) (blue and orange spectra), by following the resonant strategy described previously and sampling delays of 1 ps along the $T_{1}$ and $T_{2}$ dimensions. In Fig. 2(c), we compare the 1D spectra obtained by two-pulse ISRS in the absence of the Raman pulse, probed immediately after the photoexcitation [Fig. 2(c), blue spectrum] and after relaxation from the FC region [Fig. 2(c), orange spectrum, averaged over 300 fs along $T_{1}$, which correspond to the $A^{*}$ excited-state Raman spectrum. The comparison shows that the "fingerprint" Raman band positions up to $1200 \mathrm{~cm}^{-1}$ are not affected by the FC relaxation on $A^{*}$. This observation is in line with previous observations suggesting that the proton motion leading to the formation of the $\mathrm{I}^{*}$ form is not dominant in the GFP subpicosecond transient dynamics [61].

As a consequence of the negligible changes in the chromophore configuration during the initial relaxation process, the corresponding 2D-ISRS spectrum, shown in Fig. 3(a), presents pronounced peaks along the principal diagonal at $\Omega_{1}=\Omega_{2}$ [Fig. 3(a), black dashed diagonal line] that match frequencies obtained in the corresponding excited-state A* Raman spectrum [compare to Fig. 2(c), orange spectrum]. In addition, we observe several prominent off-diagonal peaks that occur only along vertical lines [i.e., parallel to $\Omega_{1}$, black dotted vertical lines in Fig. 3(a)], while horizontal correlations (parallel to $\Omega_{2}$ ) appear to be missing. Off-diagonal peaks are gathered mainly in three regions: a vertical stripe for $\Omega_{2}=1147 \mathrm{~cm}^{-1}$ and two subdiagonals for $\Omega_{1}=\Omega_{2}-1010 \mathrm{~cm}^{-1}$ [Fig. 3(a), red arrow] and $\Omega_{1}=\Omega_{2}-2294 \mathrm{~cm}^{-1}$ [Fig. 3(a), blue arrow]. The spectral separation between the ground-state absorption, stimulated emission, and excited-state absorption guarantees the absence of ground-state contamination to the excited-state 2D maps. This result is further confirmed by the lack of contributions of ground-state Raman frequencies in the measured 2D spectra.

\section{Origin of the 2D-ISRS couplings within a harmonic model}

The interpretation of 2D spectra is generally hampered by similar spectral features that may arise from different and concurring physical processes [62]. In order to extract the structural information contained in these measurements, we derive the 2D-ISRS signal using a perturbative framework based on the density matrix expansion [63]. We then use the obtained analytical expression (see Eq. (D1) in the Appendix) to fit the experimental data, as shown in Fig. 3(b). Further details on the signal derivation are 
(a)

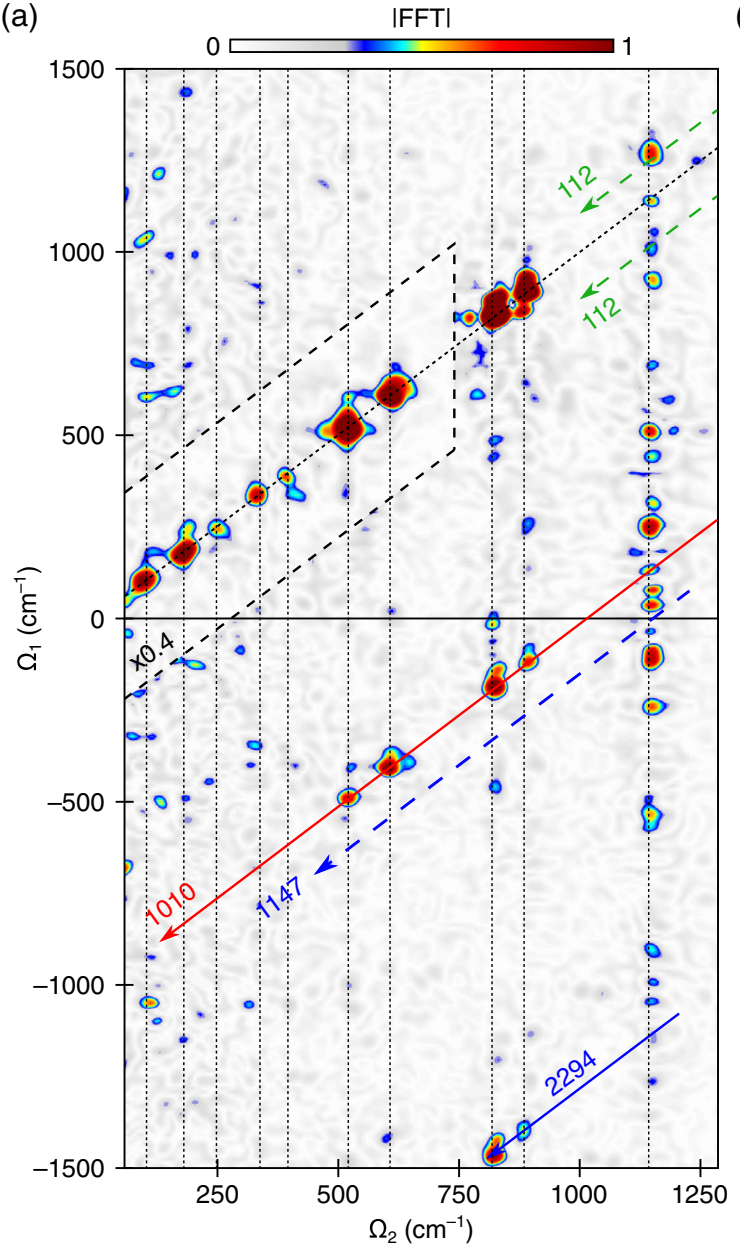

(b)

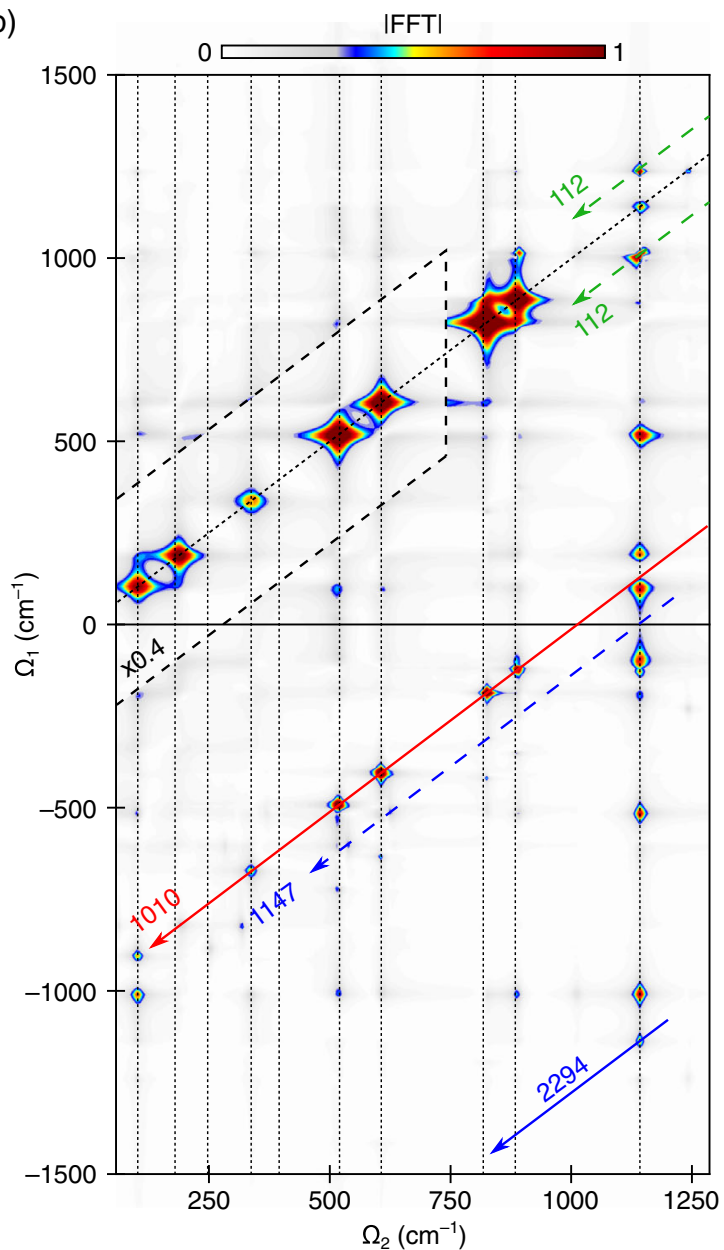

FIG. 3. The 2D-ISRS characterization of the first excited electronic state of GFP. (a) Experimental 2D-ISRS map and (b) fit to the linearly displaced harmonic model. Vertical dotted lines correspond to frequencies of the GFP excited-state vibrations, extracted by fitting the principal diagonal. The blue dashed and blue solid arrows highlight combination bands involving the fundamental and overtone frequencies of the $1147-\mathrm{cm}^{-1}$ mode, respectively. As evidenced by the experimental map in panel (a), several couplings with the overtone are present, while there are no peaks on the blue dashed arrow. The red arrow highlights combination bands involving the mode at $1010 \mathrm{~cm}^{-1}$ that is absent along the principal diagonal. The green dashed arrow highlights a combination band between the 1147 - and $112-\mathrm{cm}^{-1}$ modes. The dashed area around the principal diagonal has been scaled by 0.4 for visual purposes.

presented in the Appendix D, and in Secs. c and d of the SM [54]. Briefly, the physical observables can be related to the $n$th order nonlinear optical polarization $P^{(n)}$, which consists of the convolution between matter correlation functions and the electromagnetic fields [63]. The radiation-matter interaction is treated perturbatively, and the density matrix is expanded in power of the fields, applying many-body Green function techniques in Liouville space. Diagrammatic representations are exploited to isolate all the relevant terms in the expansion and calculate nonequilibrium expectation values of the correlation functions $[64,65]$. In particular, 2D-ISRS signals originating from the fifth-order nonlinear polarization $P^{(5)}$ are represented by the Feynman diagrams shown in Fig. 4(a).

The 2D-ISRS signal depends parametrically on the frequencies $\omega_{i}$ and lifetimes $\gamma_{i}$ of the modes and the dipole matrix elements $\mu_{i j}$, in a way determined by the choice of the model molecular Hamiltonian, $H_{0}$. As $H_{0}$ is selected, these parameters can be quantitatively computed by fitting the experimental observations to the chosen model. To describe the experimental data, we assume the harmonic oscillator (HO) approximation for $H_{0}$, in which the vibrational manifold associated with each electronic state can be depicted as an $n$-dimensional parabola. The dipole matrix elements are given by the FC overlap integrals between the initial and final wave functions, whose PESs are displaced along the normal mode coordinates with respect to each other. It is worth stressing that deviations from the harmonic regime manifest themselves mainly in offresonance conditions, when the harmonic contribution vanishes, allowing us to pinpoint the otherwise smaller contribution of single-mode and multimode anharmonicities and polarizability nonlinearities [42]. Conversely, under resonant conditions, the harmonic response is dominant. 

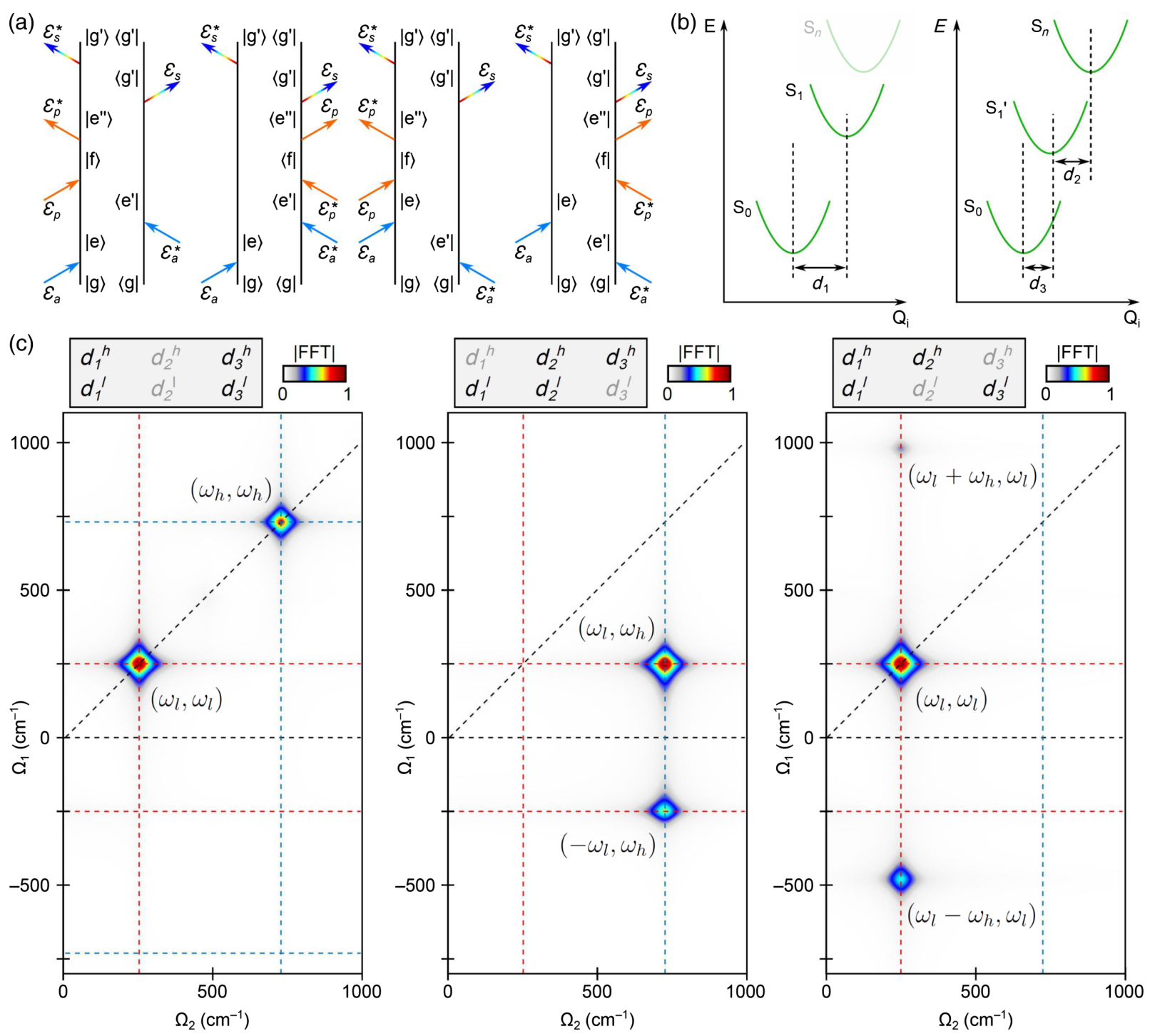

FIG. 4. Theoretical interpretation of 2D-ISRS signals in the harmonic model. (a) Double-sided Feynman diagrams describing the contributions to the signal. (b) Different energy landscapes along the vibrational coordinate $\mathrm{Q}_{i}$ probed by the actinic pulse (left panel) and Raman and probe pair (right panel). In the harmonic model, even if three electronic energy potentials are involved, three different displacements among them have to be considered, since $d_{1}$ can be different from $d_{3}$, due to the relaxation of the molecule out of the FC region. (c) Simulation of the linearly displaced harmonic model for a two-mode system $\omega_{h}=730 \mathrm{~cm}^{-1}$ and $\omega_{l}=250 \mathrm{~cm}^{-1} \mathrm{considering}$ three different choices of the displacement set, in which two of the six parameters are set equal to zero. For each panel, the two nondisplaced coordinates are indicated in gray by the color scheme on top. The left panel shows diagonal peaks obtained for $d_{2}^{h}, d_{2}^{l}=0$ and the other parameters different from zero; the central panel shows two cross peaks at $\Omega_{1}= \pm \omega_{l}, \Omega_{2}=\omega_{h}$ obtained by switching off $d_{1}^{h}$ and $d_{3}^{l}$; in the right panel, the only vanishing displacements are $d_{2}^{l}$ and $d_{3}^{h}$, which lead to a diagonal peak at $\omega_{l}$ and two combination bands at $\Omega_{1}=\omega_{h} \pm \omega_{l}$ and $\Omega_{2}=\omega_{l}$. Red (blue) horizontal and vertical lines highlight the $\omega_{l}\left(\omega_{h}\right)$ frequency.

To connect the structural information with the 2D-ISRS maps, we first consider a linearly displaced harmonic oscillator (LDHO) model with two vibrational modes $\omega_{l}$ and $\omega_{h}$ and three electronic manifolds. Within this minimal scenario, two displacements per vibrational mode are required to determine the 2D-ISRS signal. The effect of each displacement $d_{i}$ along the mode $\omega_{i}$ is to enhance or suppress a given component of the FC progression relative to that mode, gradually shifting the maximum from the $0 \rightarrow 0$ transition to higher overtones. Thus, the signal depends on the four displacements between the electronic surfaces along each vibrational coordinate $d_{1}^{h, l}$ and $d_{2}^{h, l}$. The displacement $d_{1}$ determines the probability of a transition to a specific vibrational state after the interaction with the actinic or the probe pulse since the two laser fields are resonant with the same electronic transition $\mathrm{S}_{0}-\mathrm{S}_{1}$. We remark that the fifth-order signal is emitted as a result of a six-wave mixing process upon an additional light-matter interaction (usually called free induction decay), which is due to the relaxation of the nonequilibrium polarization 
created by the external pulses. Despite the different nature of the two interactions involving the probe-pulse modes, shown as rainbow-colored arrows in Fig. 4(a), the dipole moment associated with the free induction decay is still governed by $d_{1}$, which gives the probability for a relaxation to a specific vibrational level of the electronically ground state. Similarly, $d_{2}$ controls the probability of a transition to a determined state mediated by the Raman pulse, involving the different electronic resonance $\mathrm{S}_{n}-\mathrm{S}_{1}$. From the expressions of the FC integrals, we can determine the role of each parameter: $d_{1}^{i} \neq 0$ implies FC activity of mode $i$, which can be excited by the actinic pulse and probed by the WLC as a diagonal peak in the map. In order to observe combination peaks, both $d_{1}$ and $d_{2}$ of the involved modes must be finite.

In real systems, excited-state dynamics often modify this simple scenario, even in the case of the LDHO picture. For example, in the presence of a dynamic shift of the vibrational frequencies due to coupling to a thermal bath, peaks are asymmetrically broadened with respect to the principal diagonal $[44,66]$. Here, we consider a different situation in which the dynamics are exhausted within the finite duration of the femtosecond pulses. In this case, the displacements recorded by the actinic pulse are different from those recorded by the probe pulse, as shown by Fig. 4(b), and three displacements per mode are required to appropriately describe the 2D-ISRS spectra. For these reasons, 2D-ISRS is able to also access dynamics that are too fast to be resolved as a shift in the Raman peaks or that do not modify the frequencies of the modes but only the transition dipole moments. A common situation is that upon photoexcitation by the actinic pulse, the system evolves to a different region of the $S_{1}$ PES, and the harmonic potential describing the PES near the new minimum is shifted from the initial value corresponding to $S_{1}^{\mathrm{FC}}$ to a new value corresponding to a potential $S_{1}^{\prime}$, which we refer to as the dynamic LDHO. We thus described the subpicosecond dynamics of GFP through the dynamic shift of the $S_{1}$ potential induced by the actinic pulse, in line with the observed Stokes shift [see Fig. 2(b)], and we fitted the experimental data with the dynamic LDHO model [Fig. 3(b)]. Notably, at odds with the simple picture for the static two-mode LDHO described above, off-diagonal peaks are possible even if $d_{1}$ or $d_{3}$ is vanishing for one of the modes. This case is shown in Fig. 4(c), in which we present the simulated 2D-ISRS signal of the dynamic LDHO depending on three displacements for the three typical contributions that may appear in the 2D maps: diagonal peaks $\left(\omega_{i}, \omega_{i}\right)$, cross peaks $\left(\omega_{i}, \omega_{j}\right)$, and combination peaks $\left(\omega_{i} \pm \omega_{j}, \omega_{i}\right)$. The corresponding dominant Liouville pathways are reported in Sec. e of the SM [54]. Specifically, setting two of the six available parameters equal to zero, it is possible to understand the effect of each displacement. A vanishing $d_{2}$ along both the vibrational cordinates results in diagonal peaks at the two vibrational frequencies $\omega_{l}$ and $\omega_{h}$ [Fig. 4(c), left panel]. Cross peaks between the two modes arise from switching off $d_{1}$ for one mode and $d_{3}$ for the other. For example, the cross peaks at $\Omega_{1}= \pm \omega_{l}, \Omega_{2}=\omega_{h}$ in the central panel of
Fig. 4(c) can be obtained with $d_{1}^{h}=0$ and $d_{3}^{l}=0$. Finally, combination bands at $\Omega_{1}=\omega_{h} \pm \omega_{l}=\omega_{ \pm}$and $\Omega_{2}=\omega_{l}$ as shown in the right panel of Fig. 4(c) are isolated by setting $d_{3}^{h}$ and $d_{2}^{l}$ equal to zero.

Building on such considerations based on the dynamic LDHO model, we can summarize the conditions that generate the different contributions in the 2D-ISRS maps:

(i) A diagonal peak at $\left(\omega_{i}, \omega_{i}\right)$ results from $d_{1}^{i}, d_{3}^{i} \neq 0$.

(ii) A cross peak at $\left( \pm \omega_{i}, \omega_{j}\right)$ indicates that $d_{2}$ is not vanishing, and it is comparable for the two modes.

(iii) A combination peak at $\left(\omega_{i} \pm \omega_{j}, \omega_{i}\right)$ implies a nonvanishing and comparable $d_{1}$ for the two modes. Similarly, a combination band at $\left(\omega_{i}, \omega_{i} \pm \omega_{j}\right)$ implies a nonvanishing and comparable $d_{3}$. Both additionally require $d_{2}^{j} \neq 0$.

In general, all these processes contribute to the total signal, with weights depending on the relative magnitude of the displacements. Since the signal emission requires the state of the density matrix after the free induction decay to be diagonal (see Sec. c of the SM [54] and Ref. [63]), an offdiagonal 2D-ISRS peak is obtained if the interaction with at least one of the pulses changes the total vibrational quantum number $n$ by two so that $\Delta n=\sum_{i}\left|\Delta n_{i}\right|=2$. For the cross and combination peaks analyzed above, this process is mediated by the Raman and the actinic pulses, respectively. Even if, in general, many vibrational modes are present, correlations between more than two modes are negligible in the limit of small displacements because they involve multiple higher-order processes with $\Delta n>1$. Thus, the scheme we built for the two-mode LDHO can be applied to multimode systems by considering all possible pairs.

Finally, we note that, by exploring more than two different electronic surfaces, resonant 2D-ISRS is sensitive to the sign of displacements, a key advantage over lower-order 1D techniques only capable of resolving their magnitude. Indeed, if only one electronic transition is probed (for instance, $S_{0} \rightarrow S_{1}$ ), the signal is totally symmetric with respect to a change of the sign of $d_{1}$. This result happens because, even if transitions with an odd difference of quantum numbers in the initial and final states scale linearly with the displacement, the displacement appears squared in the signal since two transitions between the same states are required to go back to a diagonal state of the density matrix. Probing an additional excited state breaks this symmetry. Even if no change occurs when all the excited-state potentials are displaced in the same direction, a different sign between pairs of $d_{i}$ and $d_{j}$ will modify the spectrum. Linear and also thirdorder techniques usually probe resonances between two electronic states, while resonant 2D-ISRS is able to specifically probe three PESs at the same time.

\section{DISCUSSION}

Building on the results of the LDHO model, we can now interpret the 2D-ISRS signal of GFP. The peaks shown in the experimental map and retrieved by the theoretical 
simulation (Fig. 3) can be classified according to the three families of diagonal, combination, and cross peaks presented in the previous section. For example, we interpret the feature at $\left(\Omega_{1}=-424 \mathrm{~cm}^{-1}, \Omega_{2}=822 \mathrm{~cm}^{-1}\right)$ as a combination band between the 822 and $1248 \mathrm{~cm}^{-1}$ modes, indicating that $d_{1}^{1248}, d_{1}^{822}$, and $d_{2}^{1248} \neq 0$. Here, we discuss the implications of the predominant features in the 2D map, while we refer to Sec. f of the SM [54] for a table summarizing the origin of all the diagonal and off-diagonal bands obtained by the fit. The presence of off-diagonal peaks along vertical and diagonal rather than horizontal lines suggests that combination peaks at $\left(\omega_{i} \pm \omega_{j}, \omega_{i}\right)$ are primarily observed and, consequently, that most of the modes are displaced along $d_{1}$ since combination bands require a nonvanishing $d_{1}$ for both of the modes involved in the signal generation. The $1010-$ $\mathrm{cm}^{-1}$ mode shows high FC activity on the $\mathrm{S}_{n}$ state, testified by the subdiagonal at $\Omega_{1} \approx \Omega_{2}-1010 \mathrm{~cm}^{-1}$ (Fig. 3, red arrow), which corresponds to a series of combination bands with other peaks due to its large $d_{2}$. In contrast, the absence of any features at $1010 \mathrm{~cm}^{-1}$ on the principal diagonal shows that $d_{3}=0$ for this mode. These observations illustrate the capability of 2D-ISRS of uncovering dark or weak bands by boosting small FC displacements via another transition.

We further observe coupling between the $1147-\mathrm{cm}^{-1}$ phenolic $\mathrm{C}-\mathrm{H}$ bend and a low-frequency mode at about $112 \mathrm{~cm}^{-1}$ (Fig. 3, green dashed arrow), which has recently been under discussion fueled by results from femtosecond stimulated Raman measurements on GFP in both the frequency and time domain [21,61]. While the functional importance of this coupling for the ESPT is under debate, the observation of oscillatory modulation of the excitedstate Raman spectrum is generally attributed to anharmonic vibrational coupling. Conversely, related studies on other molecules suggest that anharmonic couplings are challenging to isolate and interpret in fifth-order experiments due to competitive cascade processes [51]. Our study instead highlights that the coupling between the 1147and $112-\mathrm{cm}^{-1}$ modes can also be explained within a harmonic model by the displacement of the excited-state PES along these vibrational coordinates, as supported by the agreement between the combination band in our 2D measurements and fit. We further note that the intensity of this combination band is stronger above the principal diagonal than below, suggesting additional contributions due to a cross peak between the $1147-\mathrm{cm}^{-1}$ and the $1248-\mathrm{cm}^{-1}$ mode. Within our framework, single coupling peaks in the 2D map do not necessarily imply a functional importance for the ESPT reaction coordinate, but the determination of the displacements and PES orientations retrieved from the 2D-ISRS features allows for identifying the coordinates more involved in the relaxation during the first picosecond after the photoexcitation. In the case of GFP, this timescale involves the relaxation of the molecular structure towards the optimal geometry to support the ESPT [61]. In particular, the values of the displacements for the mode at $1010 \mathrm{~cm}^{-1}$ point to a relaxation of the system upon photoexcitation along this normal coordinate, which is initially in an out-ofequilibrium configuration $\left(d_{1} \neq 0\right)$ and fully relaxed at the end $\left(d_{3}=0\right)$.

Beyond the $112-\mathrm{cm}^{-1}$ mode, we observe additional couplings of the $\mathrm{C}-\mathrm{H}$ phenol mode at $1147 \mathrm{~cm}^{-1}$ (Fig. 3, vertical dotted line at $1147 \mathrm{~cm}^{-1}$ ). According to the model discussed so far, the series of peaks along the vertical at $\Omega_{2}=1147 \mathrm{~cm}^{-1}$ indicates a large value of $d_{1}$ and $d_{3}$ for this mode, while the absence of a subdiagonal at $\Omega_{1} \approx \Omega_{2}-1147 \mathrm{~cm}^{-1}$ points to a small value of $d_{2}$. Critically, the subdiagonal $\Omega_{1}=\Omega_{2}-2294 \mathrm{~cm}^{-1}$ (highlighted by the blue solid arrow in Fig. 3 ) is not captured by this model. Within the LDHO model, these features would indicate a strong $d_{2}$ displacement for the $1147-\mathrm{cm}^{-1}$ mode, such that both the fundamental and the overtone become FC active on the $\mathrm{S}_{n}$ state. This hypothesis is, however, at odds with the absence of any peaks for $\Omega_{1}=$ $\Omega_{2}-1147 \mathrm{~cm}^{-1}$ in the measured map [Fig. 3(a), blue dashed arrow].

A possible explanation of this discrepancy is the presence of a mode mixing between high- and low-frequency modes in the excited state, arising in the presence of two electronic states that have different equilibrium geometries with nonparallel corresponding normal modes, commonly denoted as Duschinsky rotation [67-69]. Under such circumstances, the normal coordinates on the excited state $\left(Q^{\prime}\right)$ can be expressed as a function of the ground state $(Q)$ as $Q^{\prime}=J Q+D$, where $J$ is the orthogonal Duschinsky matrix which depends on the rotation angles $\Theta_{\mathrm{D}}$ and $D$ is the displacement vector. Even if the excited state is not displaced, the transition $0 \rightarrow n$ with $n \neq 0$ can be strong, if the mode is coupled by the Duschinsky rotation $(J \neq \mathbb{I})$ to a displaced one. In a similar way, the FC activity of a displaced mode can decrease due to the coupling to other modes.

In order to test this hypothesis, we evaluate the 2D-ISRS response of GFP incorporating in the model a mixing between the $1147-\mathrm{cm}^{-1}$ and $80-\mathrm{cm}^{-1}$ modes, with a fixed $\Theta_{\mathrm{D}}=90^{\circ}$ Duschinsky angle. In particular, by fitting the experimental data considering the modes at $80,822,888$, and $1147 \mathrm{~cm}^{-1}$, which are those involved in the generation of the off-diagonal peaks at $\Omega_{1}=$ $\Omega_{2}-2294 \mathrm{~cm}^{-1}$, we are able to reproduce the experimental features missing in the LDHO model, as shown in Figs. 5(a) and 5(b). It is now worth dissecting the implications of the Duschinsky mechanism on the relative intensities between the fundamental and overtone contributions to combination bands. To this aim, we isolate the effect of Duschinsky rotation in Fig. 5 by considering the minimal scenario of a single mode $\left(1147 \mathrm{~cm}^{-1}\right)$, coupled to a nondisplaced low-frequency mode $\left(80 \mathrm{~cm}^{-1}\right)$, detected on a "spectator" mode $\left(822 \mathrm{~cm}^{-1}\right)$. In the absence of any 
(a)

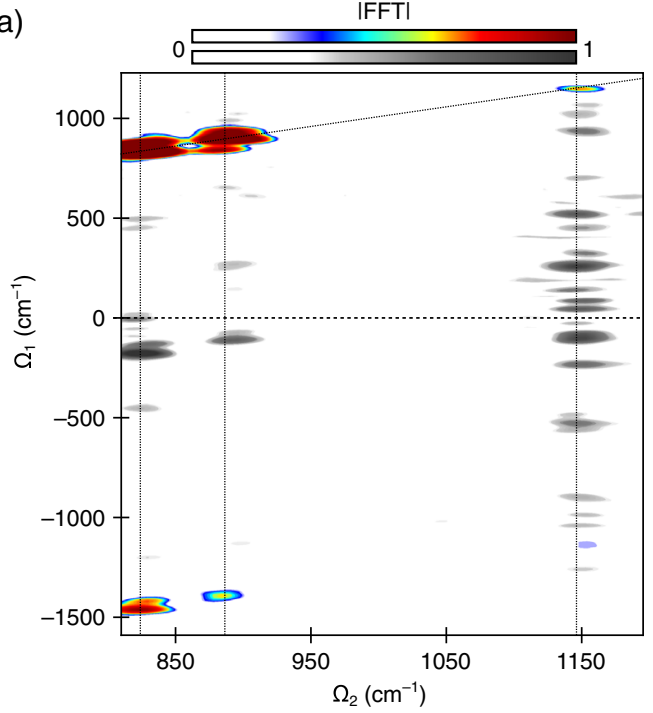

(c) $\Theta_{D}=0^{\circ}$
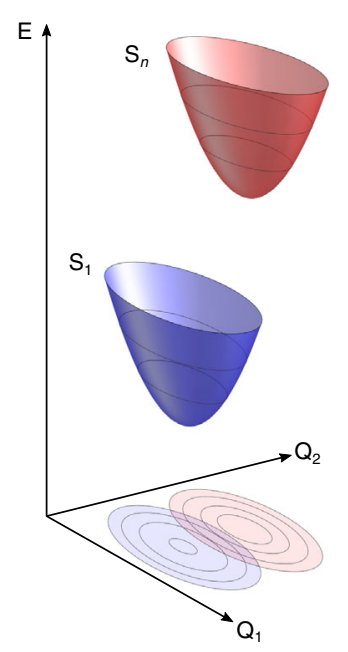

(d)

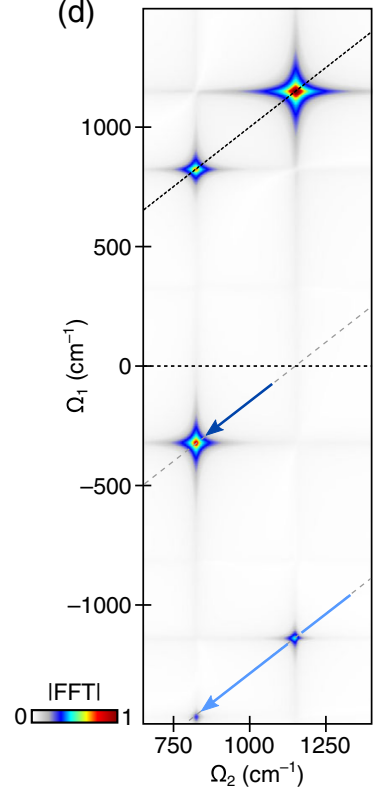

(b)

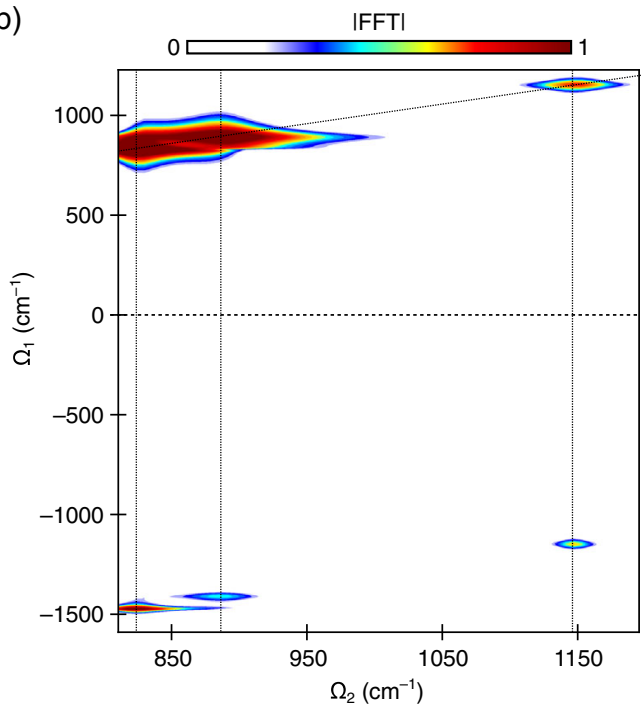

(e)

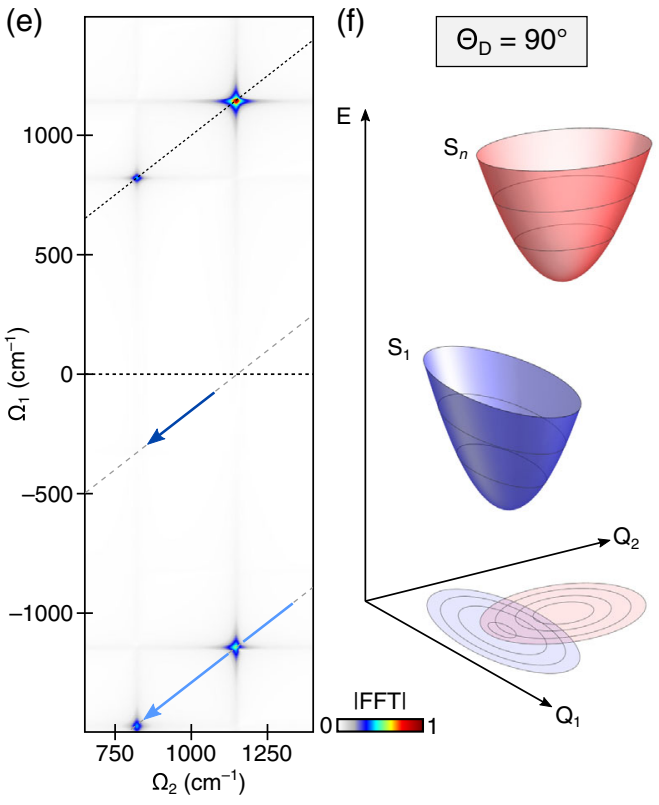

FIG. 5. Effect of mode mixing in 2D-ISRS for a harmonic model. The experimental spectra of GFP (a) are compared with the fit (b) obtained by considering the modes at $80,822,888$, and $1147 \mathrm{~cm}^{-1}$, which are those relevant for the off-diagonal peaks at $\Omega_{1}=\Omega_{2}-2294 \mathrm{~cm}^{-1}$. These features, not captured by the LDHO model, are explained by a Duschinsky rotation of $\Theta_{\mathrm{D}}=90^{\circ}$ between the 1147 - and $80-\mathrm{cm}^{-1}$ modes, and they are highlighted by the rainbow color bar in the experimental map. The gray scale has been used for the peaks not relevant for these processes and already assigned by the LDHO model. In the bottom panels, the effect of the Duschinsky rotation is dissected by considering the minimal scenario of a single mode $\left(1147 \mathrm{~cm}^{-1}\right)$, coupled to a nondisplaced lowfrequency mode $\left(80 \mathrm{~cm}^{-1}\right)$ and detected on a "spectator" mode $\left(822 \mathrm{~cm}^{-1}\right)$. For $\Theta_{\mathrm{D}}=0^{\circ}(\mathrm{a}, \mathrm{b})$, the system is described by a linearly displaced harmonic model, and a displacement $d_{2}^{1147} \neq 0$ causes the combination bands involving this mode and its overtones. The blue and cyan arrows indicate the position of combination bands associated with $1147 \mathrm{~cm}^{-1}$ and $2 \times 1147 \mathrm{~cm}^{-1}$, respectively. (c,d) In the presence of a Duschinsky rotation $\Theta_{\mathrm{D}}=90^{\circ}$ between the projections of $S_{1}$ and $S_{n}$ along the normal coordinates of the 1147- and $80-\mathrm{cm}^{-1}$ modes, the combination band associated with the fundamental of the $1147 \mathrm{~cm}^{-1}$ is suppressed.

mixing, i.e., for a vanishing Duschinsky angle $\Theta_{\mathrm{D}}=0$, $d_{2}^{1147} \neq 0$ is needed to observe coupling with the overtone of the 1147- $\mathrm{cm}^{-1}$ mode, leading to the two subdiagonals at $\Omega_{1}=\Omega_{2}-1147 \mathrm{~cm}^{-1}$ and $\Omega_{1}=\Omega_{2}-2294 \mathrm{~cm}^{-1}$ [Figs. 5(c) and 5(d), blue and cyan arrows, respectively]. Conversely, a rotation of $\Theta_{\mathrm{D}} \neq 0^{\circ}$ between projections of the excited states $S_{1}$ and $S_{n}$ along the 1147 - and $80-\mathrm{cm}^{-1}$ modes leads to a suppression of the subdiagonal peak at the fundamental frequency $\Omega_{1}=\Omega_{2}-1147 \mathrm{~cm}^{-1}$ and enhances the intensity of the overtone couplings [Figs. 5(e) and 5(f)]. The suppression is maximized at $\Theta_{D}=90^{\circ}$ for these values of the parameters and is above 
$65 \%$ for all the values of $\Theta_{\mathrm{D}}$ in the range $65^{\circ}-115^{\circ}$ (see Fig. S4 of the SM [54]).

We note that the correct identification of the origin of all the off-diagonal peaks - and, in particular, the assignment of peaks involving the overtone of the $1147 \mathrm{~cm}^{-1}$-is required to prevent wrong interpretations of the spectra; more importantly, it demonstrates the sensitivity of this technique to the Duschinsky mixing. Since the Duschinsky rotation affects the relative weights between vibronic transitions, mode mixing has a large impact on the kinetics and efficiency of charge transfer processes [70-72], making 2D-ISRS particularly suitable for studying this class of samples.

\section{CONCLUSIONS}

We introduced a resonant 2D-ISRS technique for multidimensional Raman spectroscopy. By implementing a three-color experimental scheme, we demonstrated how to decipher the correlation between vibronic modes in electronically excited molecules with electronic-state selectivity, using a perturbative sum-over-states approach. Specifically, we showed that resonant 2D-ISRS is sensitive to such correlations also in the absence of anharmonicities and efficiently suppresses lower-order cascade signals, which affect other fifth-order Raman techniques. Moreover, using a WLC probe enabled us to further isolate the electronic states from which 2D-ISRS features originate, allowing us to study the vibrational manifolds on electronically excited states. Upon identifying the vibronic origin of each peak in 2D-ISRS maps, we elucidated how different mechanisms such as linear displacements along specific normal coordinates and mode mixing contribute to the experimental signal. Notably, while lower-order techniques can only record one-dimensional projections of potential energy surfaces, 2D-ISRS is able to efficiently map complex PESs, determining FC overlaps directly over multiple dimensions.

As a proof of concept for the experimental scheme and theoretical model, we applied the technique to study the subpicosecond FC relaxation in GFP. We revealed that high-frequency mode correlations can be sustained even by fully harmonic interactions and showed that the model is capable of reproducing the previously observed coupling of the 112 - and $1147-\mathrm{cm}^{-1}$ modes. In particular, we found large FC activity on the excited state of a mode that is dark in the stimulated emission transitions and an enhancement of the mode overtones, which we linked to the presence of Duschinsky mixing. In the presence of ultrafast dynamics on the electronically excited state, the selectivity of 2DISRS can be exploited to directly access the structural conformation on the state in which the dynamics originate, disclosing the initial stages of the reaction. We anticipate that comparing mode displacements between electronic states involved for the reactant and product may be relevant to identify the key reaction coordinates implied at different stages of the photodynamics.

\section{ACKNOWLEDGMENTS}

C. S. acknowledges financial support by the Royal Commission for the Exhibition of 1851. G. Bat. acknowledges the "Avvio Alla Ricerca 2018" grant by Sapienza Universitá di Roma. T. W. acknowledges the Marie Curie Intra-European Fellowship (PIEF-GA-2013-623651) within the 7th European Community Framework Programme. S. M. gratefully acknowledges the support of the National Science Foundation Grant No. CHE-1663822.

\section{APPENDIX A: SAMPLE PREPARATION}

Plasmids containing wild-type GFP were transformed in an E. coli BL21(DE3) cell line [73]. Briefly, $0.5 \mu \mathrm{l}$ of plasmid were added in $25 \mu \mathrm{l}$ of competent BL21(DE3) cells and incubated on ice for $25 \mathrm{~min}$, heat shocked for $45 \mathrm{~s}$ at $42^{\circ} \mathrm{C}$, and incubated further for $2 \mathrm{~min}$ on ice, followed by the addition of $150 \mu \mathrm{l}$ of prewarmed at $37^{\circ} \mathrm{C}$ super optimal broth with catabolite repression media and incubation at $37^{\circ} \mathrm{C}$ with shaking for one hour. In a Lysogeny broth (LB)media Petri dish supplemented with $100 \mu \mathrm{g} / \mathrm{mL}$ kanamycin, $50 \mu \mathrm{l}$ of the cell culture were plated and incubated overnight at $37^{\circ} \mathrm{C}$. A few colonies were selected and allowed to inoculate $100 \mu \mathrm{l}$ of LB media in the presence of $100 \mu \mathrm{g} / \mathrm{mL}$ of the appropriate antibiotic and allowed to grow overnight.

The overnight cell culture was used to inoculate $8 \mathrm{~L}$ $(1 \times 8 \mathrm{~L}$ in 2 -L flasks $)$ of LB media with $100 \mu \mathrm{g} / \mathrm{ml}$ antibiotic. Cells were grown at $37^{\circ} \mathrm{C}$ to an $\mathrm{OD}_{600}$ of $0.6-0.8$ upon which $0.5 \mathrm{mM}$ IPTG was added. GFP was expressed overnight at $20^{\circ} \mathrm{C}$. Cells were harvested through centrifugation at $10000 \mathrm{xg}$ for $10 \mathrm{~min}$, and the pellets were resuspended in cell lysis buffer $(300 \mathrm{mM} \mathrm{NaCl}, 50 \mathrm{mM}$ Tris, $\mathrm{pH}$ 7.4) supplemented with a protease inhibitor tablet (Roche). Cells were lysed by microfluidizer, and the cell debris were removed by centrifugation at $20000 \mathrm{xg}$ for $20 \mathrm{~min}$. In the supernatant, imidazole was added to a final concentration of $20 \mathrm{mM}$, and the first purification step was achieved by immobilized metal ion affinity chromatography (IMAC) utilizing a 20-mL IMAC column equilibrated in buffer A ( $300 \mathrm{mM} \mathrm{NaCl}, 20 \mathrm{mM}$ Tris, and $20 \mathrm{mM}$ imidazole, $\mathrm{pH}$ 7.4). After four column volume washes with buffer $\mathrm{A}$, the sample was eluded with buffer B $(300 \mathrm{mM} \mathrm{NaCl}, 20 \mathrm{mM}$ Tris, and $500 \mathrm{mM}$ imidazole, $\mathrm{pH}$ 7.4). The sample was concentrated to a final volume of $10 \mathrm{~mL}$ and loaded in a size exclusion chromatography (SEC) column 26/60 Superdex 75 previously equilibrated in SEC buffer $(150 \mathrm{mM} \mathrm{NaCl}$ and $20 \mathrm{mM}$ Tris, $\mathrm{pH}$ 7.4). The selected fractions were pooled and concentrated to the final $\mathrm{OD}_{397}=9$.

During the spectroscopy experiments, the sample $(\sim 10 \mathrm{ml})$ was continuously flowed through a $200-\mu \mathrm{m}$ path-length flowcell at sufficient speed to ensure sample 
replenishment during consecutive shots. To minimize thermal degradation, the sample reservoir was ice cooled.

\section{APPENDIX B: EXPERIMENTAL SETUP}

All pulses were derived from a $\mathrm{Yb}: \mathrm{KGW}$ amplifier laser system (Pharos, Lightconversion) providing 5-W, 180-fs pulses centered at $1030 \mathrm{~nm}$ at a $10-\mathrm{kHz}$ repetition rate. The Raman pulse [800 nm, 9 fs, $160 \mathrm{~nJ}$, sample beam diameter of $55-\mu \mathrm{m}$ full width at half maximum (FWHM)] was generated by a noncollinear optical parametric amplifier (NOPA) pumped by the second harmonic of the laser $(515 \mathrm{~nm})$ [74]. The pulse energy was adjusted to keep twophoton absorption of ground-state molecules below $1 \%$. The actinic pulse $(400 \mathrm{~nm}, \sim 15 \mathrm{fs}, 150 \mathrm{~nJ}$, sample beam diameter of $80-\mu \mathrm{m}$ FWHM) was generated by frequency doubling the output of a second Raman NOPA $(800 \mathrm{~nm}, 10$ fs, $6 \mu \mathrm{J})$ in a $25-\mu \mathrm{m}$ beta-barium-borate crystal $\left(\theta=29^{\circ}\right)$ placed near the sample. The fundamental was removed by two reflective harmonic separators (Eksma Optics). Probe pulses (sample beam diameter: 35- $\mu \mathrm{m}$ FWHM) were generated via WLC generation in a 3-mm sapphire crystal and detected in a home-built single-shot prism spectrograph [27]. The actinic and Raman pulses were modulated at 2.5 and $5.0 \mathrm{kHz}$ by mechanical choppers, respectively, to remove lower-order contributions to the signal. $\mathrm{T}_{1}$ and $\mathrm{T}_{2}$ were sampled in 10.1- and 5.36-fs steps (ThorlabsLNR50S/M and PhysikInstrumente-M-230.10, respectively). The exact step size for each translation stage was determined by reference ISRS measurements on toluene with the same pulse parameters. All pulses were vertically polarized.

\section{APPENDIX C: DATA ANALYSIS}

The data analysis is analogous to previously reported time-domain Raman studies involving an actinic and Raman pulse [30]. Briefly, for each time point along $T_{2}$, we record three wavelength-resolved transient absorption maps as a function of $T_{2}$, corresponding to the Ramanpulse-induced differential absorbance in the presence and absence of the actinic pulse as well as the actinic-pulseinduced differential absorbance in the absence of the Raman pulse. For all three transient absorption maps, we discard time delays prior to $\mathrm{T}_{2}=100 \mathrm{fs}$, due to cross- phase modulation [75,76], and describe the electronic background by a global fit consisting of a sum of three exponential decay functions to isolate the underlying vibrational coherence. For each probe wavelength, we subsequently construct the two-dimensional signal $S\left(T_{1}, T_{2}\right)$ and apply a 2D mono-exponentially modified Gaussian window function [23-fs rise time (sigma), 1526-fs decay constant in $T_{2}$, and 6-fs rise time (sigma), 422-fs decay constant in $\mathrm{T}_{1}$ ] prior to zero padding and $2 \mathrm{D}$ fast Fourier transformation. To selectively probe only $\mathrm{S}_{1}$ vibrational coherences, we only included data greater than $536 \mathrm{fs}$ in $\mathrm{T}_{2}$, thereby suppressing additional signals due to higher-lying excited electronic states. Importantly, both time delays covered a total of $1 \mathrm{ps}$ to avoid possible distortions upon Fourier transformation. The final 2D-ISRS map is generated by averaging all detected probe wavelengths over the red side of the stimulated emission.

The FC spectrum [Fig. 2(c), blue] was obtained by Fourier transformation of the vibrational coherence in the absence of the Raman pulse. The A* spectrum [Fig. 2(c), orange] was generated by first subtracting the Raman-probe vibrational coherences in the absence of the actinic pulse from the vibrational coherences in its presence prior to Fourier transformation along $\mathrm{T}_{2}$ to yield the signal $S\left(T_{1}, \Omega_{2}\right)$ and averaging over the first $300 \mathrm{fs}$ along $\mathrm{T}_{1}$. The 2D-ISRS Fourier map in Fig. 3(a) was generated by applying the same subtraction procedure for consistency. We remark, however, that the ground-state contributions do not evolve along $T_{1}$ in the absence of the actinic pulse, resulting in similar 2D-ISRS maps with and without a ground-state subtraction procedure.

\section{APPENDIX D: SIGNAL DERIVATION}

We consider, as a reference model, a three-electroniclevel system with a ground state and two excited electronic states, $\mathrm{S}_{0}, \mathrm{~S}_{1}$, and $\mathrm{S}_{n}$, as depicted in Fig. 1(c), with the associated vibrational manifolds. The total field $\mathcal{E}$ acting on the sample consists in the three delayed pulses described above.

The 2D-ISRS signal is given by a convolution between the field amplitudes and the matter correlation function $\mathcal{F}$, derived from the four Feynman pathways shown in Fig. 4(a):

$$
\begin{aligned}
S^{(5)}\left(\omega, T_{1}, T_{2}\right)= & \mathbf{I m}\left[\left(-\frac{i}{\hbar}\right)^{5} \int_{-\infty}^{\infty} d t e^{i \omega t} \mathcal{E}_{s}^{*}(\omega) \int_{-\infty}^{t} \int_{-\infty}^{t_{5}} \int_{-\infty}^{t_{4}} \int_{-\infty}^{t_{3}} \int_{-\infty}^{t_{2}} d t_{5} \ldots d t_{1} \mathcal{E}_{a}\left(t_{1}\right) \mathcal{E}_{a}^{*}\left(t_{2}\right)\right. \\
& \left.\times \mathcal{E}_{p}\left(t_{3}-T_{1}\right) \mathcal{E}_{p}^{*}\left(t_{4}-T_{1}\right) \mathcal{E}_{s}\left(t_{5}-T_{2}-T_{1}\right) \mathcal{F}\left(t, t_{1}, t_{2}, t_{3}, t_{4}, t_{5}\right)\right] .
\end{aligned}
$$

Note that $\mathcal{F}$ can be expanded as a sum over states (SoS) [64] corresponding to the eigenfunctions of free molecule Hamiltonian $H_{0}$, obtaining 


$$
\begin{aligned}
S^{(5)}\left(\omega, T_{1}, T_{2}\right)= & \sum_{\substack{g, d^{\prime}, f \\
e, e^{\prime}, e^{\prime \prime}}} K(g) \mathbf{I m}\left\{\left[\frac{\mu_{g^{\prime} e^{\prime \prime}} \mu_{g^{\prime} e^{\prime}}^{*} \mathcal{E}_{s}^{*}(\omega) \mathcal{E}_{s}\left(\omega-\omega_{e^{\prime \prime} e^{\prime}}\right)}{2\left(\omega-\tilde{\omega}_{e^{\prime \prime} g^{\prime}}\right)}-\frac{\mu_{g^{\prime} e^{\prime \prime}} \mu_{e^{\prime} g^{\prime}}^{*} \mathcal{E}_{s}(\omega) \mathcal{E}_{s}^{*}\left(\omega+\omega_{e^{\prime \prime} e^{\prime}}\right)}{2\left(\omega+\tilde{\omega}_{g^{\prime} e^{\prime}}\right)}\right]\right. \\
& \left.\times e^{-i \tilde{\omega}_{e e^{\prime}} T_{1}} e^{-i \tilde{\omega}_{e^{\prime \prime} e^{\prime}} T_{2}} \mu_{e^{\prime \prime} f} \mu_{f e}^{*} \mathcal{W}_{p}\left(\omega_{e^{\prime \prime} e^{\prime}}-\omega_{e e^{\prime}}, \tilde{\omega}_{e^{\prime \prime} e^{\prime}}-\tilde{\omega}_{f e^{\prime}}\right) \mu_{e^{\prime} g} \mu_{e g}^{*}\left[\mathcal{W}_{a}\left(\omega_{e e^{\prime}}, \tilde{\omega}_{e e^{\prime}}-\tilde{\omega}_{e g}\right)-\mathcal{W}_{a}\left(\omega_{e e^{\prime}}, \tilde{\omega}_{g e^{\prime}}\right)\right]\right\}
\end{aligned}
$$

where $\tilde{\omega}_{i j}=\omega_{i}-\omega_{j}-i \Gamma_{i j}, \mu_{i j}$ are the matrix elements of the dipole operator $V$, and the weighted pulse spectral densities $\mathcal{W}_{k}$ for $k=a, p$ are defined as

$$
\mathcal{W}_{k}\left(\omega_{1}, \tilde{\omega}_{2}\right)=\int_{-\infty}^{+\infty} d \omega^{\prime} \frac{\mathcal{E}_{k}\left(\omega^{\prime}+\omega_{1}\right) \mathcal{E}_{k}^{*}\left(\omega^{\prime}\right)}{2\left(\omega^{\prime}+\tilde{\omega}_{2}\right)}
$$

and rule the resonant bandwidth accessible by the finite widths of the actinic and Raman pulses. The subscripts $e$, $e^{\prime}, e^{\prime \prime}$ run over the vibrational levels of $\mathrm{S}_{1}$, while $f$ and $g, g^{\prime}$ run over those of $S_{n}$ and $S_{0}$, respectively. The initial thermal population is ruled by the Boltzman factor $K(g)$ that depends on the temperature. At room temperature, vibrational modes above a few hundred wave numbers are initially populated only in the vibrational ground state; i.e., $g$ in Eq. (D1) is fixed. The displacements $\mathrm{d}_{n}, n=1,2,3$, and the Duschinsky angle $\Theta_{D}$ enter in the signal expression via the dipole matrix elements $\mu_{i j}$ as detailed in Sec. $\mathrm{d}$ of the SM [54]. Specifically, we note that the functional dependence of the signal on each displacement is the same since the signal is proportional to a product of six dipoles and each $\mathrm{d}_{n}$ appears in two of them. Finally, a Fourier transformation over the two delays and the integration on $\omega$ lead to the 2D frequency correlation map:

$$
\begin{aligned}
S^{(5)}\left(\Omega_{1}, \Omega_{2}\right)= & \int_{-\infty}^{+\infty} d \omega \\
& \times \int_{-\infty}^{+\infty} d T_{1} d T_{2} e^{i \Omega_{1} T_{1}+i \Omega_{2} T_{2}} S^{(5)}\left(\omega, T_{1}, T_{2}\right) .
\end{aligned}
$$

Using a WLC probe, the effective domain of integration over $\omega$ is restricted by the bandwidth of the pulse $\mathcal{E}(\omega)$. In our experimental implementation, the measurement is spectrally resolved in $\omega$, and a selective average over a specific spectral region can be exploited to isolate the resonant contributions from the stimulated emission or the excited-state absorption of the system. In particular, here the signal has been averaged over the tail of the stimulated emission region of the GFP, from 575 to $615 \mathrm{~nm}$, while the corresponding maps averaged on the excited-state absorption are presented in Fig. S2 of the SM [54].

In the SoS picture, the signal is determined by the contributions originating from different pathways in the Liouville space, which corresponds to the different permutation of the SoS indexes. In this way, the contribution of selected modes to the signal can be easily isolated, overcoming interpretative issues due to interference between different nonlinear optical effects, in addition to giving the advantage of speeding up the calculations by removing the contributions to an unobserved region of the spectrum. In the simulations, we did not include the contributions to the signal lying on one of the two axes, originating from pathways in which populations instead of coherences evolve during $\mathrm{T}_{1}$ or $\mathrm{T}_{2}$, since they provide the same information of third-order techniques and are suppressed by the experimental analysis routine. Further details on the signal derivation are provided in Secs. c and $d$ of the SM [54].

[1] J. C. Polanyi and A. H. Zewail, Direct Observation of the Transition State, Acc. Chem. Res. 28, 119 (1995).

[2] G. D. Scholes, G. R. Fleming, L. X. Chen, A. AspuruGuzik, A. Buchleitner, D. F. Coker, G. S. Engel, R. van Grondelle, A. Ishizaki, D. M. Jonas, J. S. Lundeen, J. K. McCusker, S. Mukamel, J. P. Ogilvie, A. Olaya-Castro, M. A. Ratner, F. C. Spano, K. B. Whaley, and X. Zhu, Using Coherence to Enhance Function in Chemical and Biophysical Systems, Nature (London) 543, 647 (2017).

[3] F. D. Fuller, J. Pan, A. Gelzinis, V. Butkus, S. S. Senlik, D. E. Wilcox, C. F. Yocum, L. Valkunas, D. Abramavicius, and J. P. Ogilvie, Vibronic Coherence in Oxygenic Photosynthesis, Nat. Chem. 6, 706 (2014).

[4] X. Gong, O. Voznyy, A. Jain, W. Liu, R. Sabatini, Z. Piontkowski, G. Walters, G. Bappi, S. Nokhrin, O. Bushuyev, M. Yuan, R. Comin, D. McCamant, S. O. Kelley, and E. H. Sargent, Electron-Phonon Interaction in Efficient Perovskite Blue Emitters, Nat. Mater. 17, 550 (2018).

[5] J. Zhou, W. Yu, and A. E. Bragg, Structural Relaxation of Photoexcited Quaterthiophenes Probed with Vibrational Specificity, J. Phys. Chem. Lett. 6, 3496 (2015).

[6] G. Batignani, E. Pontecorvo, C. Ferrante, M. Aschi, C. G. Elles, and T. Scopigno, Visualizing Excited-State Dynamics of a Diaryl Thiophene: Femtosecond Stimulated Raman Scattering as a Probe of Conjugated Molecules, J. Phys. Chem. Lett. 7, 2981 (2016).

[7] M. Cho, Coherent Two-Dimensional Optical Spectroscopy, Chem. Rev. 108, 1331 (2008).

[8] D. M. Jonas, Two Dimensional Femtosecond Spectroscopy, Annu. Rev. Phys. Chem. 54, 425 (2003). 
[9] P. Hamm and M. Zanni, Concepts and Methods of 2D Infrared Spectroscopy (Cambridge University Press, Cambridge, England, 2012).

[10] M. Fayer, Dynamics of Liquids, Molecules, and Proteins Measured with Ultrafast 2D IR Vibrational Echo Chemical Exchange Spectroscopy, Annu. Rev. Phys. Chem. 60, 21 (2009).

[11] S.-H. Shim and M. T. Zanni, How to Turn Your PumpProbe Instrument into a Multidimensional Spectrometer: 2D IR and Vis Spectroscopies via Pulse Shaping, Phys. Chem. Chem. Phys. 11, 748 (2009).

[12] A. Halpin, P. J. M. Johnson, R. Tempelaar, R. S. Murphy, J. Knoester, T. L. C. Jansen, and R. J. D. Miller, TwoDimensional Spectroscopy of a Molecular Dimer Unveils the Effects of Vibronic Coupling on Exciton Coherences, Nat. Chem. 6, 196 (2014).

[13] N. H. C. Lewis and G. R. Fleming, Two-Dimensional Electronic-Vibrational Spectroscopy of Chlorophyll $a$ and $b$, J. Phys. Chem. Lett. 7, 831 (2016).

[14] T. L. Courtney, Z. W. Fox, K. M. Slenkamp, and M. Khalil, Two-Dimensional Vibrational-Electronic Spectroscopy, J. Chem. Phys. 143, 154201 (2015).

[15] I. A. Finneran, R. Welsch, M. A. Allodi, T. F. Miller, and G. A. Blake, Coherent Two-Dimensional TerahertzTerahertz-Raman Spectroscopy, Proc. Natl. Acad. Sci. U.S.A. 113, 6857 (2016).

[16] D. B. Turner, K. W. Stone, K. Gundogdu, and K. A. Nelson, Three-Dimensional Electronic Spectroscopy of Excitons in GaAs Quantum Wells, J. Chem. Phys. 131, 144510 (2009).

[17] M. Grechko, T. Hasegawa, F. D'Angelo, H. Ito, D. Turchinovich, Y. Nagata, and M. Bonn, Coupling between Intra- and Intermolecular Motions in Liquid Water Revealed by Two-Dimensional Terahertz-Infrared-Visible Spectroscopy, Nat. Commun. 9, 885 (2018).

[18] A. P. Spencer, W. O. Hutson, and E. Harel, Quantum Coherence Selective 2D Raman-2D Electronic Spectroscopy, Nat. Commun. 8, 14732 (2017).

[19] E. Harel, Four-Dimensional Coherent Electronic Raman Spectroscopy, J. Chem. Phys. 146, 154201 (2017).

[20] T. Buckup and J. Léonard, Multidimensional Vibrational Coherence Spectroscopy, Top. Curr. Chem. 376, 35 (2018).

[21] T. Fujisawa, H. Kuramochi, H. Hosoi, S. Takeuchi, and T. Tahara, Role of Coherent Low-Frequency Motion in Excited-State Proton Transfer of Green Fluorescent Protein Studied by Time-Resolved Impulsive Stimulated Raman Spectroscopy, J. Am. Chem. Soc. 138, 3942 (2016).

[22] D. P. Hoffman, S. R. Ellis, and R. A. Mathies, Characterization of a Conical Intersection in a Charge-Transfer Dimer with Two-Dimensional Time-Resolved Stimulated Raman Spectroscopy, J. Phys. Chem. A 118, 4955 (2014).

[23] D. T. Valley, D. P. Hoffman, and R. A. Mathies, Reactive and Unreactive Pathways in a Photochemical Ring Opening Reaction from 2D Femtosecond Stimulated Raman, Phys. Chem. Chem. Phys. 17, 9231 (2015).

[24] Z. Zhang, A. Huerta-Viga, and H.-S. Tan, Two-Dimensional Electronic-Raman Spectroscopy, Opt. Lett. 43, 939 (2018).

[25] C. Ferrante, E. Pontecorvo, G. Cerullo, M. H. Vos, and T. Scopigno, Direct Observation of Subpicosecond Vibrational Dynamics in Photoexcited Myoglobin, Nat. Chem. 8, 1137 (2016).
[26] A. Kahan, O. Nahmias, N. Friedman, M. Sheves, and S. Ruhman, Following Photoinduced Dynamics in Bacteriorhodopsin with 7-fs Impulsive Vibrational Spectroscopy, J. Am. Chem. Soc. 129, 537 (2007).

[27] M. Liebel, C. Schnedermann, T. Wende, and P. Kukura, Principles and Applications of Broadband Impulsive Vibrational Spectroscopy, J. Phys. Chem. A 119, 9506 (2015).

[28] A. J. Musser, M. Liebel, C. Schnedermann, T. Wende, T. B. Kehoe, A. Rao, and P. Kukura, Evidence for Conical Intersection Dynamics Mediating Ultrafast Singlet Exciton Fission, Nat. Phys. 11, 352 (2015).

[29] C. Schnedermann, M. Liebel, and P. Kukura, ModeSpecificity of Vibrationally Coherent Internal Conversion in Rhodopsin During the Primary Visual Event, J. Am. Chem. Soc. 137, 2886 (2015).

[30] T. Wende, M. Liebel, C. Schnedermann, R. J. Pethick, and P. Kukura, Population-Controlled Impulsive Vibrational Spectroscopy: Background- and Baseline-Free Raman Spectroscopy of Excited Electronic States, J. Phys. Chem. A 118, 9976 (2014).

[31] H. Kuramochi, S. Takeuchi, and T. Tahara, Femtosecond Time-Resolved Impulsive Stimulated Raman Spectroscopy Using Sub-7-fs Pulses: Apparatus and Applications, Rev. Sci. Instrum. 87, 043107 (2016).

[32] H. Kuramochi, S. Takeuchi, K. Yonezawa, H. Kamikubo, M. Kataoka, and T. Tahara, Probing the Early Stages of Photoreception in Photoactive Yellow Protein with Ultrafast Time-Domain Raman Spectroscopy, Nat. Chem. 9, 660 (2017).

[33] M. Maiuri, E. E. Ostroumov, R. G. Saer, R. E. Blankenship, and G. D. Scholes, Coherent Wavepackets in the FennaMatthews-Olson Complex Are Robust to ExcitonicStructure Perturbations Caused by Mutagenesis, Nat. Chem. 10, 177 (2018).

[34] L. Monacelli, G. Batignani, G. Fumero, C. Ferrante, S. Mukamel, and T. Scopigno, Manipulating Impulsive Stimulated Raman Spectroscopy with a Chirped Probe Pulse, J. Phys. Chem. Lett. 8, 966 (2017).

[35] G. Batignani, G. Fumero, A. R. S. Kandada, G. Cerullo, M. Gandini, C. Ferrante, A. Petrozza, and T. Scopigno, Probing Femtosecond Lattice Displacement upon Photo-Carrier Generation in Lead Halide Perovskite, Nat. Commun. 9, 1971 (2018).

[36] G. Batignani, C. Ferrante, G. Fumero, and T. Scopigno, Broadband Impulsive Stimulated Raman Scattering Based on a Chirped Detection, J. Phys. Chem. Lett. 10, 7789 (2019).

[37] P. Kukura, D. W. McCamant, and R. A. Mathies, Femtosecond Stimulated Raman Spectroscopy, Annu. Rev. Phys. Chem. 58, 461 (2007).

[38] E. Pontecorvo, C. Ferrante, C. G. Elles, and T. Scopigno, Spectrally Tailored Narrowband Pulses for Femtosecond Stimulated Raman Spectroscopy in the Range 330-750 nm, Opt. Express 21, 6866 (2013).

[39] Z. Piontkowski and D. W. McCamant, Excited-State Planarization in Donor-Bridge Dye Sensitizers: Phenylene versus Thiophene Bridges, J. Am. Chem. Soc. 140, 11046 (2018).

[40] C. Ferrante, G. Batignani, G. Fumero, E. Pontecorvo, A. Virga, L. C. Montemiglio, G. Cerullo, M. H. Vos, and 
T. Scopigno, Resonant Broadband Stimulated Raman Scattering in Myoglobin, J. Raman Spectrosc. 49, 913 (2018).

[41] Y. Tanimura and S. Mukamel, Two-Dimensional Femtosecond Vibrational Spectroscopy of Liquids, J. Chem. Phys. 99, 9496 (1993).

[42] A. Tokmakoff, M. J. Lang, D. S. Larsen, G. R. Fleming, V. Chernyak, and S. Mukamel, Two-Dimensional Raman Spectroscopy of Vibrational Interactions in Liquids, Phys. Rev. Lett. 79, 2702 (1997).

[43] D. A. Blank, L. J. Kaufman, and G. R. Fleming, Direct Fifth-Order Electronically Nonresonant Raman Scattering from $\mathrm{CS}_{2}$ at Room Temperature, J. Chem. Phys. 113, 771 (2000).

[44] B. P. Molesky, Z. Guo, T. P. Cheshire, and A. M. Moran, Perspective: Two-Dimensional Resonance Raman Spectroscopy, J. Chem. Phys. 145, 180901 (2016).

[45] Z. Guo, B. P. Molesky, T. P. Cheshire, and A. M. Moran, Elucidation of Reactive Wavepackets by Two-Dimensional Resonance Raman Spectroscopy, J. Chem. Phys. 143, 124202 (2015).

[46] H. Frostig, T. Bayer, N. Dudovich, Y. C. Eldar, and Y. Silberberg, Single-Beam Spectrally Controlled TwoDimensional Raman Spectroscopy, Nat. Photonics 9, 339 (2015).

[47] S. Mukamel, D. Healion, Y. Zhang, and J. D. Biggs, Multidimensional Attosecond Resonant X-Ray Spectroscopy of Molecules: Lessons from the Optical Regime, Annu. Rev. Phys. Chem. 64, 101 (2013).

[48] H. Kuramochi, S. Takeuchi, H. Kamikubo, M. Kataoka, and T. Tahara, Fifth-Order Time-Domain Raman Spectroscopy of Photoactive Yellow Protein for Visualizing Vibrational Coupling in its Excited State, Sci. Adv. 5, eaau4490 (2019).

[49] T. Buckup and M. Motzkus, Multidimensional TimeResolved Spectroscopy of Vibrational Coherence in Biopolyenes, Annu. Rev. Phys. Chem. 65, 39 (2014).

[50] M. Cho, D. A. Blank, J. Sung, K. Park, S. Hahn, and G. R. Fleming, Intrinsic Cascading Contributions to the Fifthand Seventh-Order Electronically Off-Resonant Raman Spectroscopies, J. Chem. Phys. 112, 2082 (2000).

[51] K. C. Wilson, B. Lyons, R. Mehlenbacher, R. Sabatini, and D. W. McCamant, Two-Dimensional Femtosecond Stimulated Raman Spectroscopy: Observation of Cascading Raman Signals in Acetonitrile, J. Chem. Phys. 131, 214502 (2009).

[52] R. Y. Tsien, The Green Fluorescent Protein, Annu. Rev. Biochem. 67, 509 (1998).

[53] A. Acharya, A. M. Bogdanov, B. L. Grigorenko, K. B. Bravaya, A. V. Nemukhin, K. A. Lukyanov, and A. I. Krylov, Photoinduced Chemistry in Fluorescent Proteins: Curse or Blessing?, Chem. Rev. 117, 758 (2017).

[54] See Supplemental Material at http://link.aps.org/ supplemental/10.1103/PhysRevX.10.011051 for the 2DISRS time-time map (a); the 2D-ISRS maps averaged on different spectral probe regions (b); details on the derivation of the signal and the harmonic approximation (c and d); Liouville pathways for the reference simulations presented in the main text (e); details on the interpretation of spectral features in the 2D-ISRS spectra of GFP (f), and the effects of the Duschinsky angle on the relative intensities of fundamental and overtone transitions (g).
[55] We note that 2D Fourier-transformed spectra depend on positive and negative frequencies, as opposed to the 1D counterpart in which frequency axes of both signs carry the same information.

[56] D. A. Blank, L. J. Kaufman, and G. R. Fleming, Fifth-Order Two-Dimensional Raman Spectra of $\mathrm{CS}_{2}$ Are Dominated by Third-Order Cascades, J. Chem. Phys. 111, 3105 (1999).

[57] B. Dunlap, K. C. Wilson, and D. W. McCamant, PhaseMatching and Dilution Effects in Two-Dimensional Femtosecond Stimulated Raman Spectroscopy, J. Phys. Chem. A 117, 6205 (2013).

[58] J. J. van Thor, Photoreactions and Dynamics of the Green Fluorescent Protein, Chem. Soc. Rev. 38, 2935 (2009).

[59] M. Di Donato, L. J. G. W. van Wilderen, I. H. M. Van Stokkum, T. C. Stuart, J. T. M. Kennis, K. J. Hellingwerf, R. van Grondelle, and M. L. Groot, Proton Transfer Events in GFP, Phys. Chem. Chem. Phys. 13, 16295 (2011).

[60] M. Chattoraj, B. A. King, G. U. Bublitz, and S. G. Boxer, Ultra-Fast Excited State Dynamics in Green Fluorescent Protein: Multiple States and Proton Transfer, Proc. Natl. Acad. Sci. U.S.A. 93, 8362 (1996).

[61] C. Fang, R. R. Frontiera, R. Tran, and R. A. Mathies, Mapping GFP Structure Evolution During Proton Transfer with Femtosecond Raman Spectroscopy, Nature (London) 462, 200 (2009).

[62] M. Kowalewski, B. P. Fingerhut, K. E. Dorfman, K. Bennett, and S. Mukamel, Simulating Coherent Multidimensional Spectroscopy of Nonadiabatic Molecular Processes: From the Infrared to the X-Ray Regime, Chem. Rev. 117, 12165 (2017).

[63] S. Mukamel, Principles of Nonlinear Optical Spectroscopy (Oxford University, New York, 1999).

[64] K. E. Dorfman, B. P. Fingerhut, and S. Mukamel, Broadband Infrared and Raman Probes of Excited-State Vibrational Molecular Dynamics: Simulation Protocols Based on Loop Diagrams, Phys. Chem. Chem. Phys. 15, 12348 (2013).

[65] G. Fumero, G. Batignani, K. E. Dorfman, S. Mukamel, and T. Scopigno, On the Resolution Limit of Femtosecond Stimulated Raman Spectroscopy: Modelling Fifth-Order Signals with Overlapping Pulses, ChemPhysChem 16, 3438 (2015).

[66] S. Mukamel and J. D. Biggs, Communication: Comment on the Effective Temporal and Spectral Resolution of Impulsive Stimulated Raman Signals, J. Chem. Phys. 134, 161101 (2011).

[67] F. Duschinsky, The Importance of the Electron Spectrum in Multi Atomic Molecules. Concerning the Franck-Condon Principle, Acta Physicochim. URSS 7, 551 (1937).

[68] H. Kupka and P. H. Cribb, Multidimensional FranckCondon integrals and Duschinsky mixing effects, J. Chem. Phys. 85, 1303 (1986).

[69] R. Gnanasekaran, Normal Modes and the Duschinsky Mixing of the Ground- and Excited-State Vibrations of the Green Fluorescent Protein Chromophore, Chem. Phys. Lett. 587, 61 (2013).

[70] G. M. Sando, K. G. Spears, J. T. Hupp, and P. T. Ruhoff, Large Electron Transfer Rate Effects from the Duschinsky Mixing of Vibrations, J. Phys. Chem. A 105, 5317 (2001). 
[71] G. Tian, S. Duan, G.-P. Zhang, W. Hu, and Y. Luo, The Effect of Duschinsky Rotation on Charge Transport Properties of Molecular Junctions in the Sequential Tunneling Regime, Phys. Chem. Chem. Phys. 17, 23007 (2015).

[72] B. Salna, A. Benabbas, J. T. Sage, J. van Thor, and P. M. Champion, Wide-Dynamic-Range Kinetic Investigations of Deep Proton Tunnelling in Proteins, Nat. Chem. 8, 874 (2016).

[73] J. T. S. Hopper et al., Detergent-Free Mass Spectrometry of Membrane Protein Complexes, Nat. Methods 10, 1206 (2013).
[74] M. Liebel, C. Schnedermann, and P. Kukura, Sub-10-fs Pulses Tunable from 480 to $980 \mathrm{~nm}$ from a NOPA Pumped by an Yb: KGW Source, Opt. Lett. 39, 4112 (2014).

[75] G. Batignani, G. Fumero, E. Pontecorvo, C. Ferrante, S. Mukamel, and T. Scopigno, Genuine Dynamics vs Cross Phase Modulation Artifacts in Femtosecond Stimulated Raman Spectroscopy, ACS Photonics 6, 492 (2019).

[76] G. Batignani, E. Pontecorvo, D. Bossini, C. Ferrante, G. Fumero, G. Cerullo, S. Mukamel, and T. Scopigno, Modeling the Ultrafast Response of Two-Magnon Raman Excitations in Antiferromagnets on the Femtosecond Timescale, Ann. Phys. (N.Y.) 531, 1900439 (2019). 